\title{
The personal, societal, and economic burden of schizophrenia in the People's Republic of China: implications for antipsychotic therapy
}

This article was published in the following Dove Press journal:

ClinicoEconomics and Outcomes Research

16 August 2013

Number of times this article has been viewed

William Montgomery'

Li Liu ${ }^{2}$

Michael D Stensland ${ }^{5}$

Hai Bo Xue ${ }^{2}$

Tamas Treuer ${ }^{4}$

Haya Ascher-Svanum ${ }^{3}$

'Eli Lilly and Company, Sydney, NSW, Australia; 'Lilly Suzhou Pharmaceutical Company, Ltd, Shanghai, People's Republic of China; ${ }^{3}$ Eli Lilly and Company, Global Health Outcomes, Indianapolis, IN, USA; ${ }^{4}$ Eli Lilly and Company, Budapest, Hungary; ${ }^{5}$ Agile Outcomes Research, Inc, Rochester, MN, USA
Correspondence: William Montgomery Eli Lilly Australia, 112 Wharf Road, West Ryde, Sydney, NSW 2II4, Australia

Tel +6I 293254335

Fax +6I 293254334

Email montgomery_bill@lilly.com
Background: This article describes the personal, societal, and economic burden attributable to schizophrenia in the People's Republic of China and highlights the potential for effective outpatient treatment to reduce this burden given recent changes in the Chinese health care system. The importance of effective antipsychotic therapy in reducing the burden of schizophrenia is also examined.

Methods: Published research on the burden, disability, management, and economic costs of schizophrenia in the People's Republic of China was examined in the context of the larger body of global research. Research written in English or Chinese and published before June 2012 was identified using PubMed, CNKI, and Wanfang Med database searches. The contribution of effective antipsychotic therapy in reducing the risk for relapse and hospitalization and improving patients' functioning is described.

Results: Schizophrenia imposes a substantial burden on Chinese society, with indirect costs accounting for the majority of the total cost. Functional impairment is high, leading to lost wages and work impairment. In the People's Republic of China, schizophrenia is the most common diagnosis among hospitalized psychiatric patients. Ongoing changes in the Chinese health care system may reduce some barriers to effective relapse prevention in schizophrenia and potentially reduce hospitalizations. The use of antipsychotics for acute episodes and maintenance treatment has been shown to decrease symptom severity and reduce the risk for relapse and hospitalization. However, discontinuing antipsychotic medication appears common and is a strong predictor of relapse. Cost-effectiveness research in the People's Republic of China is needed to examine the potential gains from improved outpatient antipsychotic treatment.

Conclusion: Schizophrenia is a very costly mental illness in terms of personal, economic, and societal burden, both in the People's Republic of China and globally. When treated effectively, patients tend to persist longer with antipsychotic treatment, have fewer costly relapses, and have improved functioning. Further research examining the long-term effects of reducing barriers to effective treatments on the societal burden of schizophrenia in the People's Republic of China is needed.

Keywords: People's Republic of China, schizophrenia, relapse, review, health care costs, antipsychotic agents

\section{Introduction}

Schizophrenia is a highly debilitating mental illness that is seen across nearly every culture and is characterized by impaired thinking, feeling, behavior, and functioning. ${ }^{1-3}$ The World Health Organization ranks schizophrenia among the top ten causes of years lost because of disability. ${ }^{4}$ Most of the research on schizophrenia has been conducted in Western countries. The purpose of this review is to examine the available literature 
on the personal, societal, and economic burden attributable to schizophrenia in the People's Republic of China, in the context of international research in this field, and to highlight the importance of effective antipsychotic therapy and relapse prevention in reducing the burden of schizophrenia in both the acute and maintenance treatment phases.

\section{Diagnosis}

The primary symptoms in schizophrenia can be broadly categorized into positive and negative symptoms. ${ }^{1,5,6}$ Positive symptoms include delusions, hallucinations, disorganized speech, and bizarre behavior; negative symptoms include flat emotions, reduced speech, and reduced goal-driven behavior. Globally, schizophrenia is usually diagnosed using either the Diagnostic and Statistical Manual of Mental Disorders, 4th Edition, Text Revision (DSM-IV-TR) ${ }^{1}$ or the International Statistical Classification of Diseases and Related Health Problems, 10th Revision (ICD-10) criteria. ${ }^{6}$ In the People's Republic of China, schizophrenia is diagnosed using the Chinese Classification of Mental Disorders, 3rd Edition, Revised (CCMD-3) criteria. ${ }^{5}$ Although not identical, diagnostic criteria for schizophrenia within the DSM-IV-TR, ICD-10, and CCMD-3 are largely similar, although CCMD-3 differs in that it requires a loss of insight during the active phase of the illness as well as significant social dysfunction. ${ }^{5}$ Both the ICD-10 and CCMD-3 require continuous symptoms for at least 1 month, rather than the 6-month duration required by the DSM-IV-TR. Regardless of the diagnostic system, implicit in the symptoms of schizophrenia are deficits in cognition, social functioning, and productive functioning. ${ }^{7,8}$

\section{Epidemiology}

Schizophrenia is found in all societies. A meta-analysis of 85 studies estimated the worldwide prevalence of schizophrenia to be $0.54 \% .^{9}$ In the People's Republic of China, a recent large-scale study estimated the 1-month prevalence rate at $0.78 \%$, with similar rates found in rural $(0.80 \%)$ and urban $(0.72 \%)$ areas. ${ }^{10}$ Other studies in the People's Republic of China have reported prevalence estimates ranging approximately from $0.2 \%$ to $2.2 \%$. $^{9,11}$ The prevalence of schizophrenia in the People's Republic of China appears to be similar to the global prevalence.

\section{Disease management in the People's Republic of China}

When reviewing global research on schizophrenia, which has been primarily generated in Western countries, any cultural, socioeconomic, and treatment modality differences present in the People's Republic of China need to be considered. The results of the largest schizophrenia observational study to date, the Worldwide-Schizophrenia Outpatient Health Outcomes study, which did not include the People's Republic of China, revealed the existence of country-level and geographic differences in the course and prognosis of schizophrenia. In general, patients living in less developed regions had a more favorable disease course: continuous remission was lowest in Northern and Southern Europe (45.7\%) and highest in North Africa and the Middle East (67.4\%); remission followed by a relapse was lowest in North Africa and the Middle East (13.6\%); and a persistent symptomatic course was lowest in East Asia (15.1\%) and highest in Southern Europe $(39.3 \%) .{ }^{12}$ These findings parallel a previous study by the World Health Organization. ${ }^{13}$ The improved outcomes in developing countries may reflect differences in extended family structures, greater social inclusion, reduced stigma, or greater employment in schizophrenia. ${ }^{14}$ Although the better prognosis in developing countries conclusion may be an oversimplification, a recent review of 23 longitudinal studies in low- to middle-income countries found a large heterogeneity in outcomes from different studies and countries and reported that a lack of treatment was consistently associated with worse outcomes, whereas accessing care was associated with improved outcomes. ${ }^{15}$ Nevertheless, the variability in outcomes across regions highlights the need to consider the culture, socioeconomics, and treatment methods within a given region or country.

The stigma associated with mental illness can have a pernicious effect on individuals with mental health problems and their families. ${ }^{16}$ In the People's Republic of China, the disruptive behaviors of individuals with schizophrenia are considered a serious cultural transgression, and the stigma may extend to the families as well as the affected individual. ${ }^{17,18}$ The family bears a greater responsibility for managing the individuals with schizophrenia in the People's Republic of China relative to many Western cultures, in which the primary responsibility often falls on the affected individual or the health care system. ${ }^{19}$ As a result, in the People's Republic of China, individuals with schizophrenia and their families may try to hide the disorder for as long as possible, creating potential barriers to receiving and adhering with mental health treatment. ${ }^{17,18}$

The health care system in the People's Republic of China has undergone substantial changes during the last 35 years. Beginning in 1978, decreased central government support and significant policy changes led to a decentralized and privatized health care system. ${ }^{19,20}$ As part of the new market- 
based economy, hospitals were encouraged to make a profit, and many mental health rehabilitation facilities dependent on government funding were closed or transformed into small-scale psychiatric hospitals. ${ }^{21}$ These economic reforms resulted in a reduction in the number of community-based and work-rehabilitation centers, particularly in less affluent rural areas, where mental health services became essentially nonexistent. Specialized psychiatric hospitals became the primary treatment setting for patients with psychiatric conditions, and very few community-based psychiatry services existed. ${ }^{18}$ In 2002, the first National Mental Health Plan was signed. It included stated goals to establish an effective system for mental health care and to strengthen existing mental health services to decrease the burden and disability associated with mental illness. Several programs were initiated, and they have had a significant effect in some regions. $^{21}$

A mental health reform program, named the 686 Program, received formal support from the Ministry of Finance in 2004. By early 2005, 60 demonstration sites were established, including one urban and one rural site in each of the People's Republic of China's 30 provinces. The program screened patients for possible psychosis, subsidized the cost of medications and hospitalizations for the socially disadvantaged, and implemented community action programs that encompassed the best-practice principles from allied health disciplines. By 2009, the program's coverage had been expanded to include 112 cities and 96.9 million people, or $7.3 \%$ of the People's Republic of China's 1.33 billion population. ${ }^{21,22}$

Large changes in the availability of health insurance have also occurred in the last 15 years in the People's Republic of China. The rapid economic growth experienced within the People's Republic of China has been concentrated in the urban areas and has contributed to regional disparities. ${ }^{23}$ Although commercial health insurance schemes that primarily target the more affluent in Chinese society have expanded, the major change has been the introduction of a countrywide health insurance system, known as basic social medical insurance (BSMI). The BSMI has expanded rapidly, driven by the central government's goal to provide universal health coverage to the entire population of the People's Republic of China. ${ }^{24}$ The BSMI consists of three components: the basic social medical insurance for urban employees (initiated in 1998), the basic social medical insurance for urban residents (initialed in 79 cities in 2007), and the New Rural Cooperative Medical Insurance for Rural Residents (established in 2003). ${ }^{24}$ The tremendous changes in insurance coverage implemented in the last decade have the potential to improve access to effective health care for hundreds of millions of people and to potentially reduce health-related disability in the People's Republic of China.

Although insurance coverage is now available to most individuals in the People's Republic of China, specific coverage for schizophrenia and mental health is varied. In some of the wealthier Eastern areas, the mental health service system is being reformed quickly within whole provinces or cities, but in some underdeveloped Western areas, where skills and resources are lacking, the existing national mental health policies have not yet been implemented. ${ }^{21}$ The implementation of specific mental health policies and services also tends to be more common in Eastern China than Western China and for urban compared with rural residents. More than $30 \%$ of the regions have no specific health insurance policies for mental disorders, and where policies do exist, they tend to be appropriately aimed at the reimbursement of outpatient services. ${ }^{25}$ Without appropriate coverage for outpatient care of patients with schizophrenia, there is a risk for inadvertently incentivizing the overuse of more expensive hospitalization and inpatient services.

Past research has also shown that many patients with schizophrenia in the People's Republic of China may not always receive needed care. A large-scale epidemiologic study in the People's Republic of China reported that $27.6 \%$ of patients with psychotic disorders never sought treatment. ${ }^{10}$ Similarly, another study in rural China found that almost one-third of individuals with schizophrenia received no treatment at all, despite $76 \%$ of these individuals having marked symptoms. ${ }^{11}$ The primary reasons reported for the patient not receiving treatment were lack of money $(35.3 \%)$, uncertainty about the patient's illness $(29.5 \%)$, the patient refusing to accept drug treatment (18.6\%), and having no relatives to care for the patient $(14.7 \%) .{ }^{26}$ Perceptions and psychological attributions of schizophrenia as well as issues of availability, access, and affordability of care may represent important barriers to effective treatment in the People's Republic of China.

Another important barrier in the People's Republic of China is the limited number of mental health care practitioners and inpatient facilities. In 2004, the People's Republic of China had 1.99 psychiatric nurses and 1.29 psychiatrists per 100,000 population compared with 6.50 and 13.70 , respectively, in the United States and 24.80 and 9.80 in Europe. Similarly, the People's Republic of China had 1.06 psychiatric beds per 10,000 population compared with 7.70 in the United States and 8.00 in Europe. ${ }^{27}$ Although there are fewer mental health care practitioners and inpatient facilities 
in the People's Republic of China, estimates of the length of stay for a patient with schizophrenia have ranged from 36.8 to 69.9 days, ${ }^{28,29}$ which is longer than the 7.4 to 11.1 days seen in the United States, ${ }^{30} 30.7$ days seen in Australia, ${ }^{31}$ and 35 days seen in Spain, ${ }^{32}$ but similar to the number of days seen in Finland. ${ }^{33}$ The longer inpatient stays in the People's Republic of China may reflect fewer available communitybased treatment options.

\section{Disability}

The World Health Organization lists schizophrenia in the top eight causes of years lost to disability. ${ }^{4,34}$ In the People's Republic of China, schizophrenia was ranked as the 18th most important health problem. ${ }^{18}$

The functional impairments associated with schizophrenia are sufficiently severe to be apparent in high-level indicators such as unemployment and marriage rates. Globally, the unemployment rate in samples of individuals with schizophrenia has ranged from $10 \%$ to $96 \%$, depending on the study method and country, with the majority of estimates being greater than $75 \% .{ }^{35-39}$ In the People's Republic of China, estimates of the unemployment rate for patients with schizophrenia have ranged from $41.3 \%$ to $64.7 \% .^{40-43}$ Although lower than many global estimates for the rate of unemployment in schizophrenia, these rates are substantially higher than the overall unemployment rate in the People's Republic of China, which is reported to be lower than in most countries. ${ }^{44}$

Global estimates of marriage rates for patients with schizophrenia have ranged from about $5 \%$ to $30 \%$, with most between $15 \%$ and $30 \% .{ }^{35-37,45-48}$ In the People's Republic of China, the rates of marriage for samples of individuals with schizophrenia have ranged from $10.9 \%$ to $46.7 \% .^{28,40-43}$ Marriage and employment rates may be slightly higher in the People's Republic of China than globally, but they remain substantially lower than in populations without schizophrenia because of the strong effect of the disorder on social and productive functioning.

\section{Economic consequences of schizophrenia}

Schizophrenia is an expensive disorder and is associated with high levels of direct costs to the health care system and very high levels of indirect costs, primarily because of lost productivity. Direct costs refer to payments that are made, including those for inpatient stays, outpatient services, and medications, whereas indirect costs refer to resources that are lost as a result of the illness, including lost wages of the patient, lost wages of caregivers, and lost years of life.
A review of multiple cost-of-illness studies for schizophrenia from around the globe reported that the direct health care costs of treating schizophrenia accounted for between $1.4 \%$ and $3.0 \%$ of total national health expenditures. ${ }^{49}$ In the People's Republic of China, schizophrenia has been reported to contribute $1.3 \%$ of the total burden of disease. ${ }^{50}$

Comparing costs across geographies is challenging, given differences in study methodologies and health care systems. Although few studies have taken a comprehensive view of the cost of schizophrenia across all of the People's Republic of China, several studies have examined the costs in specific geographic areas.

\section{Cost of schizophrenia in the People's Republic of China}

Zhai et a ${ }^{50}$ followed-up 299 patients with schizophrenia from Shandong and Hunan provinces who met DSM-IV diagnostic criteria for 1 year. The patients and their caregivers maintained receipts for all health care services and medications received and completed the Economic Cost Questionnaire every 2 months during the year. The median annual costs in Chinese currency per patient were $¥ 4370$ (approximately US \$700) in direct costs and $¥ 10,081$ (approximately US \$1600) in indirect costs. Direct costs for those patients who had at least one hospitalization ( $¥ 7614$ ) were substantially higher than for those who were not hospitalized ( $¥ 1812 ; P<0.001$ ). Caregivers bore a substantial part of the indirect costs ( $¥ 3600$ ). Although the annual per patient costs are much lower in the People's Republic of China than in most Western countries, the average net income of a rural resident was only $¥ 2936$ in 2004 ( $¥ 9422$ disposable income for an urban resident), ${ }^{51}$ and much of the indirect cost may have fallen on the patient and his or her family. ${ }^{50}$

In Zhejiang province in 2010 , other research examined the direct psychiatric hospital costs for 7684 inpatients. Schizophrenia was the most common diagnosis, making up 3117 of the 7684 discharges. Median total cost for schizophrenia was $¥ 10,765$ per admission or $¥ 241$ per day. Psychiatric medications made up $¥ 834$ of the cost during the hospital admission. Schizophrenia and other psychotic disorders had the longest duration of admission, with an average of 45 days. $^{29}$

\section{Cost of schizophrenia outside the People's Republic of China}

Administrative claims from the Taiwanese governmental health insurance data in 1999 found that the equivalent of US \$112.4 million was spent on care for schizophrenia, which 
represents $1.2 \%$ of the total national health care expenditures. Most of the expenditures (US $\$ 84.5$ million or $75.2 \%$ ) were on inpatient care, with US \$27.9 million spent on outpatient care, with inpatient and outpatient medication costs of US \$28.9 million. On a per patient basis, the average annual direct treatment costs for schizophrenia were US $\$ 2144 .^{52}$ Another study in Taiwan followed up 74 patients treated with risperidone or haloperidol for 1 year beginning during 1999 or 2000. The average annual per patient direct costs were estimated at US $\$ 2115$, but indirect costs were substantially higher, at US $\$ 14,461$, primarily because of lost wages for the patient and caregivers. ${ }^{53}$

Using data from the National Health Insurance in South Korea, the total direct health care costs for schizophrenia during 2005 were estimated at US \$418.7 million, with inpatient costs accounting for US $\$ 327.7$ million (78.3\%), outpatient costs being US $\$ 77.7$ million (18.6\%), and medication costs being US \$13.3 million (3.2\%). The average per patient annual total direct treatment cost was US \$2600. Indirect costs were substantially higher, at US \$2635.1 million, and were driven primary by lost wages. ${ }^{54}$

In comparison, the total costs in the United States in 2002 were estimated to be $\$ 62.7$ billion, with direct health care costs comprising $\$ 22.7$ billon. ${ }^{55}$ Hospital and long-term care costs made up $47 \%$, outpatient care $31 \%$, and medications $22.2 \%$ of the direct health care costs. The annual per patient direct cost was $\$ 15,464 .{ }^{55}$

The higher indirect costs reported in the People's Republic of China are consistent with findings from other geographies, although Asian countries appear to have a larger proportion of indirect costs (87\% in Taiwan, ${ }^{53} 83 \%$ in Korea, ${ }^{54}$ and $63 \%$ in India $\left.^{56}\right)$ than those reported in Western countries ( $47 \%$ to $81 \%) .{ }^{49}$ Because of substantial disability, individuals with schizophrenia incur significant indirect costs resulting from lost wages, premature mortality and suicide, ${ }^{49,55}$ involvement with the criminal justice system, ${ }^{57}$ and caregiver burden. ${ }^{49,58}$ In a review of multiple studies from various geographies, the high direct and indirect costs of schizophrenia have been consistently reported. ${ }^{49}$

\section{Health care resource use}

Globally, the use of inpatient facilities is a significant component of the cost of treating schizophrenia. ${ }^{55,59}$ In a review of cost-of-illness studies for schizophrenia, Knapp et al ${ }^{49}$ found that hospitalization accounted for between $16.5 \%$ and $94 \%$ of costs, depending on the country and study methods, with twothirds of the studies finding that hospitalization accounted for more than $50 \%$ of total direct health care costs.
Throughout the world, studies have reported the length of hospitalization for each schizophrenia admission to range from 7.4 to 90 days. ${ }^{30,60-63}$ In the People's Republic of China, published estimates of the length of hospitalization for patients with schizophrenia have ranged from 36.8 to 69.9 days, depending on the year and the health insurance status of the patient, with insured patients tending to have longer inpatient stays. ${ }^{28,29}$ These estimates may not be representative of the current situation or the whole country, given the rapidly changing health care system and regional variations in the People's Republic of China.

Medications also account for a significant, but substantially lower, proportion $(2.0 \%-13.0 \%$ in Western countries) of direct health care costs compared with inpatient and outpatient services. ${ }^{49,59}$ One study in Zhejiang province reported that the overall cost of hospitalization was $¥ 10,765$ for schizophrenia, with $¥ 834$ for psychiatric medications and $¥ 719$ for nonpsychiatric medication. ${ }^{29}$ The use of effective antipsychotic medication has been shown to decrease both inpatient and outpatient health care use, ${ }^{49}$ and low rates of medication adherence have been shown to be associated with higher total inpatient costs. ${ }^{64,65}$

\section{Treatment goals}

The treatment goals for patients with schizophrenia vary, depending on the phase of the illness. During an acute episode, the goals of treatment are to prevent harm, control disturbed behavior, suppress symptoms, attempt to get a rapid return to the best level of functioning, and develop an effective treatment alliance with the patient and family. ${ }^{2,8}$ Once in the stabilization phase, usually 3-6 months after the acute phase, the primary goals are the consolidation of the therapeutic relationship, reduction of positive symptoms, improvement in cognitive and negative symptoms, improvement of social deficits, promotion of insight and medication adherence, support of developing individual coping strategies, integration of the patient back into their community, and identification of support options to minimize the rate of relapse. ${ }^{3,8}$ Finally, in the stable phase, the goals become improvement of functioning and recovery, maintaining or improving quality of life, and prevention of relapses. ${ }^{3,8}$ Therapeutic interventions for schizophrenia include both psychosocial and pharmacological treatments, and the best course of treatment is often a combination of both. In the People's Republic of China, adding psychosocial interventions to medications has been shown to decrease the risk for medication discontinuation and relapse and to improve quality of life and functioning compared with medication alone. ${ }^{66}$ 


\section{Role of antipsychotics}

Antipsychotic medications have been the mainstay of the treatment for schizophrenia since their introduction in the 1950s and are recommended for use to treat acute psychotic states as well as maintenance treatment to prevent future relapses..$^{2,8,67,68}$ Frequently, antipsychotic medications are broadly categorized as being either typical, first-generation antipsychotics (FGAs) or the newer, atypical, secondgeneration antipsychotics (SGAs). FGAs and SGAs have been thought to differ in terms of treatment effectiveness, tolerability, and adverse event profile. ${ }^{69}$ The SGAs have been reported to lead to improvements in patient outcomes and a reduced risk for extrapyramidal adverse events compared with FGAs. ${ }^{67,70}$ However, FGAs and SGAs are heterogeneous groups of medications that have been somewhat arbitrarily grouped together primarily as a result of being developed before or after clozapine. ${ }^{69}$ In clinical care, SGAs have largely replaced the older FGAs, with risperidone, olanzapine, and quetiapine often being the most commonly prescribed agents. ${ }^{71,72}$ Research examining antipsychotic use patterns for schizophrenia in the People's Republic of China also suggests that the SGAs are replacing the FGAs. ${ }^{73}$ The most commonly used SGAs in the People's Republic of China appear to be clozapine, risperidone, olanzapine, and quetiapine. ${ }^{74}$

\section{Relapse}

Symptomatic relapse in schizophrenia can be devastating not only to the patients but also to their families. When the patients' symptoms remain under control, the patients can slowly begin to reintegrate into society; however, when they experience an acute relapse, the setback can be dramatic, often disrupting relationships and employment. Relapse often results in hospitalization ${ }^{35,63,75}$ and leads to increases in the use of outpatient services and medication use. ${ }^{75}$ Relapses tend to increase symptom persistence, disrupt efforts at rehabilitation, and damage relationships, and may lead to greater cognitive decline..$^{76,77}$

\section{Predictors of relapse}

Global treatment guidelines for schizophrenia recommend continuing antipsychotic medications for a minimum or 1 to 2 years after a first-episode patient is stable because this treatment is one of the most efficacious means of relapse prevention. ${ }^{3,8,68}$ For patients who have had multiple episodes, continuing antipsychotic treatment for a minimum of 5 years and potentially throughout the lifetime is recommended. ${ }^{3,8}$
A recent meta-analysis of 65 randomized controlled trials found that compared with those taking placebo, patients taking antipsychotic medications were significantly less likely to relapse (relative risk, 0.35 ) or to be hospitalized (relative risk, 0.38).$^{78}$ The number needed to treat indicated that during the study periods, for every three patients maintained on an antipsychotic instead of placebo, a single relapse would be prevented. ${ }^{78}$ The evidence from global studies is strong that antipsychotic maintenance treatment prevents future relapses.

Nonadherence with antipsychotic medication is one of the strongest predictors of relapse. ${ }^{79}$ When medications are discontinued, the risk for symptomatic relapse has been shown to increase by nearly five times ${ }^{80}$ Similarly, medication nonadherence has been shown to be predictive of increased hospitalization rates ${ }^{81-84}$ In the People's Republic of China, a randomized controlled trial found that relative to continuing treatment with the initial antipsychotic (risperidone) dose, random assignment to dose reduction increased the risk for relapse substantially (odds ratio, 2.9), as did poor adherence with medication. ${ }^{43}$

Nonadherence may be particularly problematic in the People's Republic of China when patients and their families do not believe that the patient needs to continue treatment with an antipsychotic medication. ${ }^{26,85}$ An epidemiologic study identified 510 patients with schizophrenia in rural Xinjin county and found that only $30(5.9 \%)$ were currently taking antipsychotic treatment, $92(18.0 \%)$ underwent inconsistent treatment for less than 2 months, 106 (20.8\%) had only used traditional Chinese herbal medicines, and 156 (30.6\%) had never received any treatment. ${ }^{11}$ A more recent observational study followed up 1133 patients treated with antipsychotics and found that $40.5 \%$ discontinued the study antipsychotic during the 1-year study ${ }^{86}$ Other reviews have reported that most patients did not remain on medication for more than 1 year after their initial hospital admission. ${ }^{18}$ More research is needed to understand the rates of early medication discontinuation and the factors that lead to early discontinuation of antipsychotic treatment in the People's Republic of China.

\section{Antipsychotics differ in effectiveness}

Global research has found that patients are more likely to continue treatment with some antipsychotics relative to others. Time to discontinuation of an antipsychotic for any cause has become an important marker of the real-world effectiveness of antipsychotics in the treatment of schizophrenia. ${ }^{87}$ Global research has found that patients will continue taking, and clinicians will continue to prescribe, an antipsychotic 
if they believe the efficacy of the medication, particularly for positive symptoms, outweighs any tolerability issues. ${ }^{88}$ A recent review of the comparative effectiveness of atypical antipsychotics for the treatment of schizophrenia found that clozapine was the most effective antipsychotic, followed by olanzapine and then by all other atypical and typical antipsychotics. ${ }^{89}$ Because of its greater efficacy, clozapine remains the treatment of choice for treatment-resistant patients, despite blood monitoring recommendations because of the potential for agranulocytosis, which can be fatal. ${ }^{8,90}$ Among other (nonclozapine) atypical antipsychotics, olanzapine has been shown to differ on effectiveness when measured as time to all-cause medication discontinuation.

The National Institute of Mental Health-sponsored Clinical Antipsychotic Trials of Intervention Effectiveness (CATIE) study found that patients persisted longer with olanzapine than quetiapine or risperidone.${ }^{91}$ Similarly, large observational trials in Europe have found patients treated with clozapine and olanzapine remain on their antipsychotic medication for a longer period of time than many other commonly prescribed atypical or typical antipsychotics. ${ }^{33,92,93}$ The only study specifically examining antipsychotic discontinuation rates in the People's Republic of China, a prospective observational study, failed to find any significant differences. ${ }^{86}$ However, the rate of treatment discontinuation at 12 months was high (36.7\%-46.9\%), and the treatment duration (mean, 8.9 months) was short for all antipsychotics studied. The cost of medications in this study was paid for by the patients or their families, and the authors state that affordability may have been a factor that influenced the results. ${ }^{86}$

The CATIE study also examined the rates of hospitalization for exacerbations of schizophrenia during the 18-month study. ${ }^{91,94}$ Similar to the findings on medication discontinuation, the risk for hospitalization was lowest for olanzapine-treated patients and highest among the quetiapine-treated patients (Figure 1).

Similarly, a large, prospective, observational study of patients with schizophrenia treated in usual clinical care in Europe also found that olanzapine provided significantly improved negative, positive, and cognitive symptoms compared with risperidone, quetiapine, oral typicals, and depot typicals..$^{95}$

\section{Antipsychotic efficacy}

In contrast to effectiveness research, which examines health outcomes from treatments when implemented in usual clinical care, efficacy research examines key clinical outcomes such as symptom reduction from protocol-implemented treatment in well controlled studies. A meta-analysis of 150 randomized double-blind controlled trials comparing the efficacy of typical and atypical antipsychotics found better overall efficacy for the atypical antipsychotics on positive and negative symptoms compared with the typical antipsychotics. When specific atypical antipsychotics

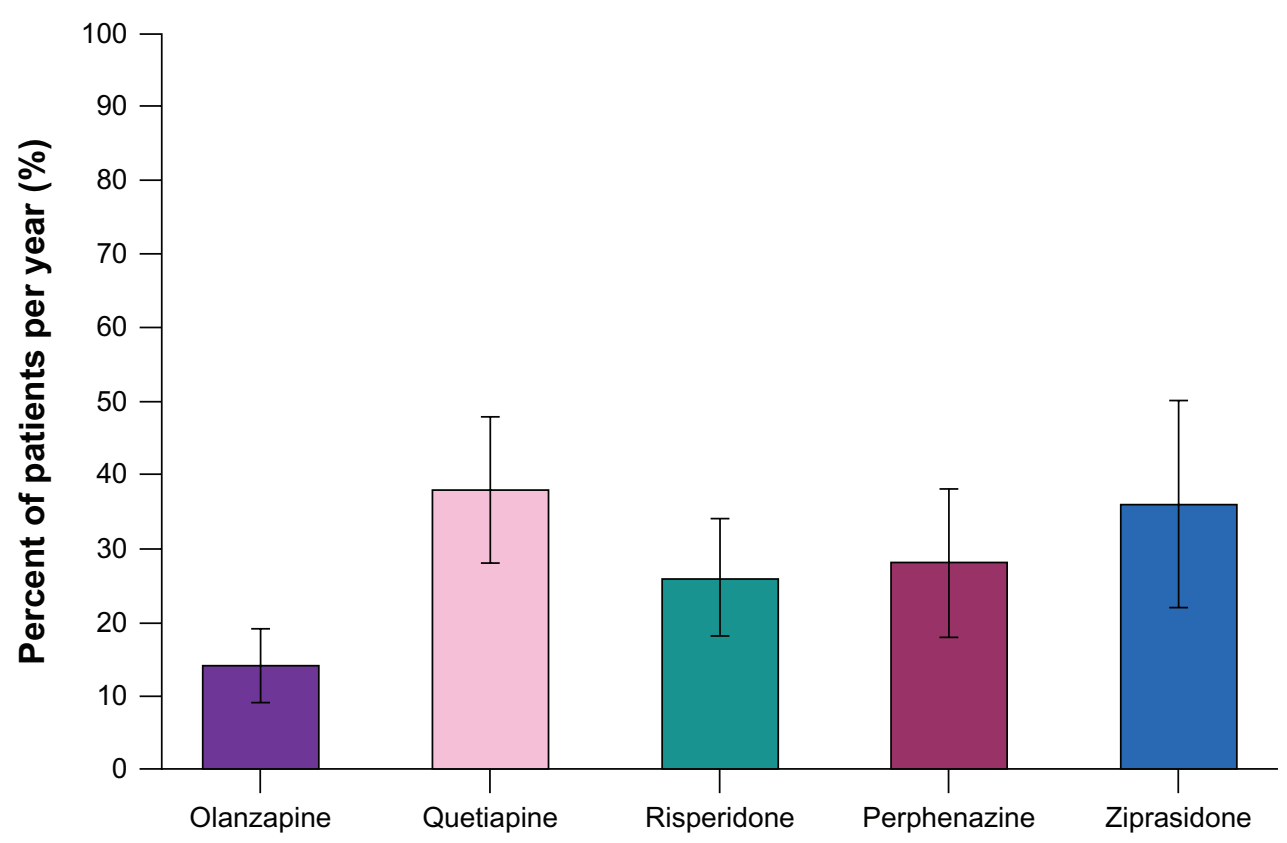

Figure I Annual hospitalization rates for exacerbation of schizophrenia in CATIE (Phase I). ${ }^{94}$ Adapted with permission from Psychiatric Services, (Copyright @20II). American Psychiatric Association. All Rights Reserved.

Notes: Error bars represent $95 \%$ confidence intervals. Olanzapine was significantly different $(P<0.05)$ from quetiapine, perphenazine, and ziprasidone. Abbreviation: CATIE, Clinical Antipsychotic Trials of Intervention Effectiveness. 
were compared with typical antipsychotics, greater efficacy was observed for clozapine, olanzapine, amisulpride, and risperidone, but not the other atypical antipsychotics. ${ }^{96}$ In another meta-analysis of 78 randomized clinical trials comparing the atypical antipsychotics head-to-head, the authors found that the atypicals could not be considered all alike on efficacy, with olanzapine showing significantly greater efficacy than risperidone, quetiapine, aripiprazole, or ziprasidone; clozapine showing significantly greater efficacy than zotepine; and risperidone showing significantly greater efficacy than quetiapine and ziprasidone. ${ }^{97}$ An examination of longer-term symptomatic relapse rates across studies found that patients taking olanzapine had significantly less risk for relapse compared with those treated with risperidone, quetiapine, ziprasidone, or typical antipsychotics. ${ }^{98}$ Globally, research has found that the short-term efficacy and longerterm relapse prevention of atypical antipsychotics varies for the different compounds, with greater efficacy reported for clozapine and olanzapine.

The Chinese literature on the comparative efficacy of antipsychotics appears similar to the global research. In a randomized, open-label trial of antipsychotic drug-naïve Chinese patients, olanzapine demonstrated significantly greater decreases in schizophrenic symptoms compared with ziprasidone. ${ }^{99}$ A study comparing risperidone and aripiprazole reported no differences in efficacy, ${ }^{100}$ and another study showed greater efficacy of risperidone over haloperidol for treatment-resistant patients. ${ }^{101}$ The research from the People's Republic of China, although less comprehensive, appears consistent with the global research.

\section{Antipsychotics and tolerability}

A meta-analysis using randomized double-blind studies of atypical and typical antipsychotics in the treatment of schizophrenia found that patients treated with atypicals were significantly less likely to need adjunctive treatment for extrapyramidal symptoms but were significantly more likely to gain weight, particularly when treated with clozapine or olanzapine. ${ }^{96,102}$ Olanzapine treatment has also been associated with significantly higher increases in cholesterol than aripiprazole, risperidone, and ziprasidone and with significantly higher increases in blood glucose than amisulpride, aripiprazole, quetiapine, risperidone, and ziprasidone. ${ }^{102}$ Risperidone-treated patients have been found to have a significantly higher likelihood for prolactin increases and sexual dysfunction than other atypical antipsychotics. ${ }^{98,103}$ Studies conducted in the People's Republic of China have also found significantly reduced extrapyramidal symptoms, ${ }^{86,104}$ but greater weight gain and greater increases in cholesterol and triglycerides, ${ }^{86,104-106}$ for olanzapine-treated patients relative to patients treated with risperidone, ziprasidone, or typical antipsychotics. Both global and Chinese studies have found that efficacy and tolerability profiles vary for different antipsychotics. The clinician's goal is to identify the antipsychotic with the efficacy and tolerability profile best suited to a specific individual with schizophrenia.

Effective management of adverse events may lead to improved outcomes. A recent post hoc analysis from the Chinese subgroup $(n=330)$ of a multicountry, 6-month, prospective, observational study of outpatients with schizophrenia who were treated with olanzapine compared outcomes for patients who participated or did not participate in a weight control program. Not only did the participants in the weight control program gain less weight but they also had significantly better clinical and functional outcomes: greater improvement in illness severity, higher treatment response rate, shorter time to response, reduced impairment in work activities, and greater improvement in patients' insight. ${ }^{107,108}$ This finding supports the clinical experience that participation in a weight control program may help prevent clinically significant weight gain in patients treated with antipsychotics and may also be associated with additional clinical and functional benefits that may help prevent relapse.

\section{Discussion}

Schizophrenia imposes a high burden on Chinese society, particularly on the individuals with the disease and their families. ${ }^{50}$ The Chinese health care system has undergone major changes since 1978, becoming more decentralized and privatized. ${ }^{19-21}$ At this time, there are initiatives to establish universal health care coverage, and a growing number of individuals are covered by health insurance systems, which can provide some relief from the high direct health care costs of schizophrenia for the patient and family members. ${ }^{21,24}$ In the recent past, very few community-based psychiatric services have been available, particularly in rural areas, with most services provided by specialty psychiatric hospitals that were primarily located in urban areas. ${ }^{21}$ In late 2012, the Mental Health Law of the People's Republic of China was passed. In addition to providing more protection to people suffering from mental illness, including a new protocol for commitment to psychiatric hospitals, the new law supports the expansion of community mental health services. ${ }^{109,110}$ With the ongoing mental health reforms, there is an opportunity for the development of cost-effective outpatient treatment services that use effective medications 
to prevent relapses. In terms of direct health care costs, hospitalizations are expensive and often of long duration. ${ }^{29}$ However, the greatest burden associated with schizophrenia in the People's Republic of China is a result of indirect costs ${ }^{50}$ which include the burden on the caregiver and family and the costs associated with lost productivity, social dysfunction, and premature mortality.

Unfortunately, in the People's Republic of China, many patients with schizophrenia do not initiate or persist with antipsychotic treatment. Several barriers to receiving antipsychotic treatment exist, including patient and family beliefs about the need for continuing antipsychotics, the cost of the medications to the families, and the limited availability of outpatient services. ${ }^{26}$ This discordance between actual and recommended practice is a major cause for concern. Research has repeatedly linked antipsychotic discontinuation with greater rates of relapse and hospitalization and with further functional disability. ${ }^{78-80}$ The burden of the disease could potentially be reduced in the People's Republic of China if persistence on antipsychotic therapy could be increased.

Improved access to effective treatments in the People's Republic of China, particularly the combination of family education and antipsychotic treatment, could help reduce the personal, societal, and economic burden of this disease. One study found that adding family-based psychoeducational treatment to antipsychotic medication reduced treatment discontinuation and relapses and increased social functioning, health-related quality of life, and employment rates. ${ }^{66}$ Recent reviews of antipsychotic efficacy and effectiveness have shown that patients are more likely to persist on therapy with the more effective second-generation antipsychotics for a longer period of time..$^{89,111}$ Cost-effectiveness research investigating potential gains from improved access to effective antipsychotic treatment of people with schizophrenia in the People's Republic of China is needed, given the high costs associated with this condition to the individual, the health care system, and society.

\section{Conclusion}

Improving access and increasing the levels of persistence with antipsychotic medications in the People's Republic of China would likely reduce the rate of relapse, improve patient outcomes, and reduce total treatment costs. Several barriers exist to optimum treatment, including the paucity of outpatient psychiatric centers, beliefs about the need for maintenance treatment once acute symptoms are treated, and the relatively high cost of medication to patients and families. Increasing the duration of maintenance treatment, particularly with effective antipsychotics, would likely translate into lower rates of relapse, which is expected to help reduce the personal, societal, and economic burden of schizophrenia in the People's Republic of China by improving patient outcomes.

\section{Disclosure}

The authors report no conflicts of interest in this work. William Montgomery, Li Liu, Haya Ascher-Svanum, Hai Bo Xue, and Tamas Treuer are all full time employees of Eli Lilly and Company or one subsidiaries and minor stockholders in Eli Lilly and Company. Michael Stensland is a full time employee of Agile Outcomes Research Inc, a research firm hired to consult on this work.

\section{References}

1. American Psychiatric Association. Diagnostic and Statistical Manual of Mental Disorders, 4th Edition, Text Revision, 4th ed. Arlington, VA: American Psychiatric Association; 2000.

2. Falkai P, Wobrock T, Lieberman J, Glenthoj B, Gattaz WF, Möller HJ; for WFSBP Task Force on Treatment Guidelines for Schizophrenia. World Federation of Societies of Biological Psychiatry (WFSBP) guidelines for biological treatment of schizophrenia, Part 1: acute treatment of schizophrenia. World J Biol Psychiatry. 2005;6(3):132-191.

3. Falkai P, Wobrock T, Lieberman J, Glenthoj B, Gattaz WF, Möller HJ; for WFSBP Task Force on Treatment Guidelines for Schizophrenia World Federation of Societies of Biological Psychiatry (WFSBP) guidelines for biological treatment of schizophrenia, part 2: longterm treatment of schizophrenia. World J Biol Psychiatry. 2006;7(1) $5-40$.

4. Mathers C, Boerma T, Fat DM. The Global Burden of Disease: 2004 Update. Geneva: World Health Organization; 2008. Available from: http://www.who.int/healthinfo/global_burden_disease/GBD_ report_2004update_full.pdf. Accessed November 1, 2012.

5. Chinese Society of Psychiatry. Chinese Classification of Mental Disorders, 3rd ed. Shandong, China: Shandong Science and Technology Press; 2001.

6. World Health Organization. International Statistical Classification of Diseases and Related Health Problems, 10th Revision. Version for 2007. Geneva: World Health Organization; 2007. Available from: http://apps.who.int/classifications/apps/icd/icd10online2007/. Accessed September 19, 2012

7. Lambert M, Karow A, Leucht S, Schimmelmann BG, Naber D. Remission in schizophrenia: validity, frequency, predictors, and patients' perspective 5 years later. Dialogues Clin Neurosci. 2010;12(3):393-407.

8. Lehman AF, Lieberman JA, Dixon LB, et al; for American Psychiatric Association, Steering Committee on Practice Guidelines. Practice guideline for the treatment of patients with schizophrenia, second edition. Am J Psychiatry. 2004;161(Suppl 2):1-56.

9. Saha S, Chant D, McGrath J. Meta-analyses of the incidence and prevalence of schizophrenia: conceptual and methodological issues. Int J Methods Psychiatr Res. 2008;17(1):55-61.

10. Phillips MR, Zhang J, Shi Q, et al. Prevalence, treatment, and associated disability of mental disorders in four provinces in China during 20012005: an epidemiological survey. Lancet. 2009;373(9680): 2041-2053.

11. Ran MS, Xiang MZ, Li SX, et al. Prevalence and course of schizophrenia in a Chinese rural area. Aust N Z J Psychiatry. 2003;37(4):452-457.

12. Novick D, Haro JM, Hong J, et al. Regional differences in treatment response and three year course of schizophrenia across the world. J Psychiatr Res. 2012;46(7):856-864. 
13. Jablensky A, Sartorius N, Ernberg G, et al. Schizophrenia: manifestations, incidence and course in different cultures. A World Health Organization ten-country study. Psychol Med Monogr Suppl. 1992; 20:1-97.

14. Birchwood M, Cochrane R, Macmillan F, Copestake S, Kucharska J, Carriss $\mathrm{M}$. The influence of ethnicity and family structure on relapse in first-episode schizophrenia. A comparison of Asian, Afro-Caribbean, and white patients. Br J Psychiatry. 1992;161:783-790.

15. Cohen A, Patel V, Thara R, Gureje O. Questioning an axiom: better prognosis for schizophrenia in the developing world? Schizophr Bull. 2008;34(2):229-244.

16. Phillips MR, Pearson V, Li F, Xu M, Yang L. Stigma and expressed emotion: a study of people with schizophrenia and their family members in China. Br J Psychiatry. 2002;181:488-493.

17. Yang LH. Application of mental illness stigma theory to Chinese societies: synthesis and new directions. Singapore Med J. 2007;48(11): 977-985.

18. Phillips MR. Characteristics, experience, and treatment of schizophrenia in China. Dialogues Clin Neurosci. 2001;3(2):109-119.

19. Park L, Xiao Z, Worth J, Park JM. Mental health care in China: recent changes and future challenges. Harvard Health Policy Rev. 2005;6(2):35-45.

20. Blumenthal D, Hsiao W. Privatization and its discontents - the evolving Chinese health care system. N Engl J Med. 2005;353(11):1165-1170.

21. Liu J, Ma H, He YL, et al. Mental health system in China: history, recent service reform and future challenges. World Psychiatry. 2011;10(3): 210-216.

22. China Statistical Yearbook 2010 [homepage on the Internet]. National Bureau of Statistics in China. Beijing, China: China Statistics Press; 2010. Available from: http://www.stats.gov.cn/tjsj/ndsj/2010/indexeh. htm. August 14, 2012.

23. Hung LM, Rane S, Tsai J, Shi L. Advancing primary care to promote equitable health: implications for China. Int $J$ Equity Health. 2012;11(1):2

24. Dong K. Medical insurance system evolution in China. China Economic Review. 2009;20(4):591-597.

25. Liang D, Wang Q, Ying X. [Analysis on status quo of medical insurance policies on mental disorders in China.] China Health Policy Studies. 2011;4(7):46-50. Chinese. http://d.wanfangdata.com.cn/periodical_zgwszcyj201107010.aspx. Accessed May 20, 2013.

26. Ran M, Xiang M, Huang M, Shan Y. Natural course of schizophrenia: 2-year follow-up study in a rural Chinese community. Br J Psychiatry. 2001;178(2):154-158.

27. World Health Organization. Mental health atlas 2005 [webpage on the Internet]. Geneva: World Health Organization; 2005. Available from: http://www.who.int/mental_health/evidence/mhatlas05/en/. Accessed November 2, 2012.

28. Phillips MR, Lu SH, Wang RW. Economic reforms and the acute inpatient care of patients with schizophrenia: the Chinese experience. Am J Psychiatry. 1997;154(9):1228-1234.

29. Yang SL, Qian MC, Lu W, et al. Cost of treating medical conditions in psychiatric inpatients in Zhejiang, China. Shanghai Archives of Psychiatry. 2011;23(6):329-337.

30. Stensland M, Watson PR, Grazier KL. An examination of costs, charges, and payments for inpatient psychiatric treatment in community hospitals. Psychiatr Serv. 2012;63(7):666-671.

31. Low LF, Draper B. Hospitalization patterns for psychiatric disorders across the lifespan in Australia from July 1998 to June 2005. Psychiatr Serv. 2009;60(1):113-116.

32. Saz-Parkinson Z, Medel A, Cediel-García P, Castellote J, Bouza C, Amate JM. Trends on schizophrenia admissions during the deinstitutionalisation process in Spain (1980-2004). Soc Psychiatry Psychiatr Epidemiol. 2011;46(11):1095-1101.

33. Tiihonen J, Wahlbeck K, Lönnqvist J, et al. Effectiveness of antipsychotic treatments in a nationwide cohort of patients in community care after first hospitalisation due to schizophrenia and schizoaffective disorder: observational follow-up study. BMJ. 2006;333(7561):224
34. Rössler W, Salize HJ, van Os J, Riecher-Rössler A. Size of burden of schizophrenia and psychotic disorders. Eur Neuropsychopharmacol. 2005;15(4):399-409.

35. Almond S, Knapp M, Francois C, Toumi M, Brugha T. Relapse in schizophrenia: costs, clinical outcomes and quality of life. Br J Psychiatry. 2004;184(4):346-351.

36. Alonso J, Croudace T, Brown J, et al. Health-related quality of life (HRQL) and continuous antipsychotic treatment: 3-year results from the Schizophrenia Health Outcomes (SOHO) study. Value Health. 2009;12(4):536-543.

37. Ciudad A, Alvarez E, Bobes J, San L, Polavieja P, Gilaberte I. Remission in schizophrenia: results from a 1-year follow-up observational study. Schizophr Res. 2009;108(1-3):214-222.

38. Marwaha S, Johnson S. Schizophrenia and employment - a review. Soc Psychiatry Psychiatr Epidemiol. 2004;39(5):337-349.

39. Perlick DA, Rosenheck RA, Kaczynski R, Bingham S, Collins J. Association of symptomatology and cognitive deficits to functional capacity in schizophrenia. Schizophr Res. 2008;99(1-3):192-199.

40. Yeung FK, Chan SH. Clinical characteristics and objective living conditions in relation to quality of life among community-based individuals of schizophrenia in Hong Kong. Qual Life Res. 2006;15(9):1459-1469.

41. Xiang YT, Weng YZ, Leung CM, Tang WK, Ungvari GS. Quality of life of Chinese schizophrenia outpatients in Hong Kong: relationship to sociodemographic factors and symptomatology. Aust NZ J Psychiatry. 2007;41(5):442-449.

42. Xiang YT, Wang CY, Wang Y, et al. Socio-demographic and clinical determinants of quality of life in Chinese patients with schizophrenia: a prospective study. Qual Life Res. 2010;19(3):317-322.

43. Xiang YT, Wang CY, Weng YZ, et al. Predictors of relapse in Chinese schizophrenia patients: a prospective, multi-center study. Soc Psychiatry Psychiatr Epidemiol. 2011;46(12):1325-1330.

44. Giles J, Park A, Zhang J. What is China's true unemployment rate? China Economic Review. 2005;16(2):149-170.

45. Advokat C, Dixon D, Schneider J, Comaty JE Jr. Comparison of risperidone and olanzapine as used under "real-world" conditions in a state psychiatric hospital. Prog Neuropsychopharmacol Biol Psychiatry. 2004;28(3):487-495.

46. Ascher-Svanum H, Nyhuis AW, Faries DE, Kinon BJ, Baker RW, Shekhar A. Clinical, functional, and economic ramifications of early nonresponse to antipsychotics in the naturalistic treatment of schizophrenia. Schizophr Bull. 2008;34(6):1163-1171.

47. Haro JM, Edgell ET, Frewer P, Alonso J, Jones PB; for SOHO Study Group. The European Schizophrenia Outpatient Health Outcomes Study: baseline findings across country and treatment. Acta Psychiatr Scand Suppl. 2003;416:7-15.

48. Silva de Lima M, de Jesus Mari J, Breier A, Maria Costa A, Pondé de Sena E, Hotopf M. Quality of life in schizophrenia: a multicenter, randomized, naturalistic, controlled trial comparing olanzapine to firstgeneration antipsychotics. J Clin Psychiatry. 2005;66(7):831-838.

49. Knapp M, Mangalore R, Simon J. The global costs of schizophrenia. Schizophr Bull. 2004;30(2):279-293.

50. Zhai JG, Zhao JP, Chen JD. An investigation on economic costs of schizophrenia in two medical establishments in Hunan and Shandong. Chinese Journal of Psychiatry. 2007;40(1):41-44.

51. China Statistics Press. [National Economic and Social Development Statistics Bulletin 2004.] China Statistics http://www.stats.gov.cn/tjgb/ ndtjgb/qgndtjgb/t20050228_402231854.htm. Accessed May 20, 2013. Chinese.

52. Lang HC, Su TP. The cost of schizophrenia treatment in Taiwan. Psychiatr Serv. 2004;55(8):928-930.

53. Lee IH, Chen PS, Yang YK, et al. The functionality and economic costs of outpatients with schizophrenia in Taiwan. Psychiatry Res. 2008;158(3):306-315.

54. Chang SM, Cho SJ, Jeon HJ, et al. Economic burden of schizophrenia in South Korea. J Korean Med Sci. 2008;23(2):167-175.

55. Wu EQ, Birnbaum HG, Shi L, et al. The economic burden of schizophrenia in the United States in 2002. J Clin Psychiatry. 2005;66(9):1122-1129. 
56. Grover S, Avasthi A, Chakrabarti S, Bhansali A, Kulhara P. Cost of care of schizophrenia: a study of Indian out-patient attenders. Acta Psychiatr Scand. 2005;112(1):54-63.

57. Ascher-Svanum H, Nyhuis AW, Faries DE, Ball DE, Kinon BJ. Involvement in the US criminal justice system and cost implications for persons treated for schizophrenia. BMC Psychiatry. 2010;10:11.

58. Awad AG, Voruganti LNP. The burden of schizophrenia on caregivers: a review. Pharmacoeconomics. 2008;26(2):149-162.

59. Garattini L, Barbui C, Clemente R, Cornago D, Parazzini F; for Study Group SCORE. Direct costs of schizophrenia and related disorders in Italian community mental health services: a multicenter, prospective 1-year follow up study. Schizophr Bull. 2004;30(2):295-302.

60. Ascher-Svanum H, Zhu B, Faries D, Ernst FR. A comparison of olanzapine and risperidone on the risk of psychiatric hospitalization in the naturalistic treatment of patients with schizophrenia. Ann Gen Hosp Psychiatry. 2004;3(1):11.

61. Daltio CS, Mari JJ, Ferraz MB. Direct medical costs associated with schizophrenia relapses in health care services in the city of São Paulo. Rev de Saude Publica. 2011;45(1):14-23.

62. Gau SS, Chung CH, Gau CS. A pharmacoeconomic analysis of atypical antipsychotics and haloperidol in first-episode schizophrenic patients in Taiwan. J Clin Psychopharmacol. 2008;28(3):271-278.

63. Weiden PJ, Olfson M. Cost of relapse in schizophrenia. Schizophr Bull. 1995;21(3):419-429.

64. Eaddy M, Grogg A, Locklear J. Assessment of compliance with antipsychotic treatment and resource utilization in a Medicaid population. Clin Ther. 2005;27(2):263-272.

65. Knapp M, King D, Pugner K, Lapuerta P. Non-adherence to antipsychotic medication regimens: associations with resource use and costs. Br J Psychiatry. 2004;184(6):509-516.

66. Guo X, Zhai J, Liu Z, et al. Effect of antipsychotic medication alone vs combined with psychosocial intervention on outcomes of early-stage schizophrenia: a randomized, 1-year study. Arch Gen Psychiatry. 2010;67(9):895-904.

67. Buchanan RW, Kreyenbuhl J, Kelly DL, et al; for Schizophrenia Patient Outcomes Research Team (PORT). The 2009 schizophrenia PORT psychopharmacological treatment recommendations and summary statements. Schizophr Bull. 2010;36(1):71-93.

68. National Collaborating Centre for Mental Health. Schizophrenia: The NICE Guideline on Core Interventions in the Treatment and Management of Schizophrenia in Adults in Primary and Secondary Care (Updated edition). Leicester, UK: The British Psychological Society and the Royal College of Psychiatrists; 2010. Available from: http:/ www.nice.org.uk/nicemedia/live/11786/43607/43607.pdf. Accessed September 18, 2012.

69. Leucht S, Kissling W, Davis JM. Second-generation antipsychotics for schizophrenia: can we resolve the conflict? Psychol Med. 2009;39(10):1591-1602.

70. Dossenbach M, Erol A, el Mahfoud Kessaci M, et al; for IC-SOHO Study Group. Effectiveness of antipsychotic treatments for schizophrenia: interim 6-month analysis from a prospective observational study (IC$\mathrm{SOHO}$ ) comparing olanzapine, quetiapine, risperidone, and haloperidol. J Clin Psychiatry. 2004;65(3):312-321

71. Prah P, Petersen I, Nazareth I, Walters K, Osborn D. National changes in oral antipsychotic treatment for people with schizophrenia in primary care between 1998 and 2007 in the United Kingdom. Pharmacoepidemiol Drug Saf. 2012;21(2):161-169.

72. Weinbrenner S, Assion HJ, Stargardt T, Busse R, Juckel G, Gericke CA Drug prescription patterns in schizophrenia outpatients: analysis of data from a German health insurance fund. Pharmacopsychiatry. 2009;42(2):66-71.

73. An FR, Xiang YT, Wang CY, et al. Change of psychotropic drug prescription for schizophrenia in a psychiatric institution in Beijing, China between 1999 and 2008. Int J Clin Pharmacol Ther. 2010;48(4): 270-274.

74. Chong MY, Tan CH, Fujii S, et al. Antipsychotic drug prescription for schizophrenia in East Asia: rationale for change. Psychiatry Clin Neurosci. 2004;58(1):61-67.
75. Ascher-Svanum H, Zhu B, Faries DE, et al. The cost of relapse and the predictors of relapse in the treatment of schizophrenia. BMC Psychiatry. 2010;10:2.

76. Amminger GP, Edwards J, Brewer WJ, Harrigan S, McGorry PD. Duration of untreated psychosis and cognitive deterioration in firstepisode schizophrenia. Schizophr Res. 2002;54(3):223-230.

77. Lieberman JA, Perkins D, Belger A, et al. The early stages of schizophrenia: speculations on pathogenesis, pathophysiology, and therapeutic approaches. Biol Psychiatry. 2001;50(11):884-897.

78. Leucht S, Tardy M, Komossa K, Heres S, Kissling W, Davis JM. Maintenance treatment with antipsychotic drugs for schizophrenia. Cochrane Database Syst Rev. 2012;5:CD008016.

79. Ayuso-Gutiérrez JL, del Río Vega JM. Factors influencing relapse in the long-term course of schizophrenia. Schizophr Res. 1997;28(2-3): 199-206.

80. Robinson D, Woerner MG, Alvir JM, et al. Predictors of relapse following response from a first episode of schizophrenia or schizoaffective disorder. Arch Gen Psychiatry. 1999;56(3):241-247.

81. Gilmer TP, Dolder CR, Lacro JP, et al. Adherence to treatment with antipsychotic medication and health care costs among Medicaid beneficiaries with schizophrenia. Am J Psychiatry. 2004;161(4): 692-699.

82. Law MR, Soumerai SB, Ross-Degnan D, Adams AS. A longitudinal study of medication nonadherence and hospitalization risk in schizophrenia. J Clin Psychiatry. 2008;69(1):47-53.

83. Weiden PJ, Kozma C, Grogg A, Locklear J. Partial compliance and risk of rehospitalization among California Medicaid patients with schizophrenia. Psychiatr Serv. 2004;55(8):886-891.

84. Svarstad BL, Shireman TI, Sweeney JK. Using drug claims data to assess the relationship of medication adherence with hospitalization and costs. Psychiatr Serv. 2001;52(6):805-811.

85. Phillips MR, Li Y, Stroup TS, Xin L. Causes of schizophrenia reported by patients' family members in China. Br J Psychiatry. 2000;177: $20-25$

86. Guo X, Fang M, Zhai J, et al. Effectiveness of maintenance treatments with atypical and typical antipsychotics in stable schizophrenia with early stage: 1-year naturalistic study. Psychopharmacology (Berl). 2011;216(4):475-484

87. Stroup TS, McEvoy JP, Swartz MS, et al. The National Institute of Mental Health Clinical Antipsychotic Trials of Intervention Effectiveness (CATIE) project: schizophrenia trial design and protocol development. Schizophr Bull. 2003;29(1):15-31.

88. Ascher-Svanum H, Nyhuis AW, Stauffer V, et al. Reasons for discontinuation and continuation of antipsychotics in the treatment of schizophrenia from patient and clinician perspectives. Curr Med Res Opin. 2010;26(10):2403-2410.

89. Attard A, Taylor DM. Comparative effectiveness of atypical antipsychotics in schizophrenia: what have real-world trials taught us? CNS Drugs. 2012;26(6):491-508.

90. Lahdelma L, Appelberg B. Clozapine-induced agranulocytosis in Finland, 1982-2007: long-term monitoring of patients is still warranted. J Clin Psychiatry. 2012;73(6):837-842.

91. Lieberman JA, Stroup TS, McEvoy JP, et al; for Clinical Antipsychotic Trials of Intervention Effectiveness (CATIE) Investigators. Effectiveness of antipsychotic drugs in patients with chronic schizophrenia. N Engl J Med. 2005;353(12):1209-1223.

92. Tiihonen J, Haukka J, Taylor M, Haddad PM, Patel MX, Korhonen P. A nationwide cohort study of oral and depot antipsychotics after first hospitalization for schizophrenia. Am J Psychiatry. 2011;168(6): 603-609.

93. Haro JM, Suarez D, Novick D, Brown J, Usall J, Naber D; for SOHO Study Group. Three-year antipsychotic effectiveness in the outpatient care of schizophrenia: observational versus randomized studies results. Eur Neuropsychopharmacol. 2007;17(4):235-244.

94. Olfson M, Ascher-Svanum H, Faries DE, Marcus SC. Predicting psychiatric hospital admission among adults with schizophrenia. Psychiatr Serv. 2011;62(10):1138-1145. 
95. Haro JM, Edgell ET, Novick D, et al; for SOHO advisory board. Effectiveness of antipsychotic treatment for schizophrenia: 6-month results of the Pan-European Schizophrenia Outpatient Health Outcomes (SOHO) study. Acta Psychiatr Scand. 2005;111(3):220-231.

96. Leucht S, Corves C, Arbter D, Engel RR, Li C, Davis JM. Secondgeneration versus first-generation antipsychotic drugs for schizophrenia: a meta-analysis. Lancet. 2009;373(9657):31-41.

97. Leucht S, Komossa K, Rummel-Kluge C, et al. A meta-analysis of head-to-head comparisons of second-generation antipsychotics in the treatment of schizophrenia. Am J Psychiatry. 2009;166(2):152-163.

98. Glick ID, Correll CU, Altamura AC, et al. Mid-term and long-term efficacy and effectiveness of antipsychotic medications for schizophrenia: a data-driven, personalized clinical approach. J Clin Psychiatry. 2011; 72(12):1616-1627.

99. Wu XL, Wang JH, Hu SH, Tao J. Serum prolactin levels and the acutephase efficacy in drug-naïve schizophrenia treated with ziprasidone and olanzapine (translated version). East Asian Arch Psychiatry. 2012;22(1):7-11.

100. Chan HY, Lin WW, Lin SK, et al. Efficacy and safety of aripiprazole in the acute treatment of schizophrenia in Chinese patients with risperidone as an active control: a randomized trial. J Clin Psychiatry. 2007;68(1):29-36.

101. Zhang XY, Zhou DF, Cao LY, Zhang PY, Wu GY, Shen YC. Risperidone versus haloperidol in the treatment of acute exacerbations of chronic inpatients with schizophrenia: a randomized double-blind study. Int Clin Psychopharmacol. 2001;16(6):325-330.

102. Rummel-Kluge C, Komossa K, Schwarz S, et al. Head-to-head comparisons of metabolic side effects of second generation antipsychotics in the treatment of schizophrenia: a systematic review and metaanalysis. Schizophr Res. 2010;123(2-3):225-233.

103. Komossa K, Rummel-Kluge C, Schwarz S, et al. Risperidone versus other atypical antipsychotics for schizophrenia. Cochrane Database Syst Rev. 2011;1:CD006626.
104. Li YM, Zhao JP, Ou JJ, Wu RR. Efficacy and tolerability of ziprasidone vs olanzapine in naive first-episode schizophrenia: a 6-week, randomized, open-label, flexible-dose study. Pharmacopsychiatry. 2012;45(5):177-181.

105. Lee E, Leung CM, Wong E. Atypical antipsychotics and weight gain in Chinese patients: a comparison of olanzapine and risperidone. J Clin Psychiatry. 2004;65(6):864-866.

106. Lee E, Chow LY, Leung CM. Metabolic profile of first and second generation antipsychotics among Chinese patients. Psychiatry Res. 2011;185(3):456-458.

107. Ang Q, Tang J, Zhao J. Factors associated with weight gain during six-month olanzapine therapy. Chinese Journal of Psychiatry. 2011;44(3):1-6.

108. Montgomery W, Treuer T, Ye W, et al. Does participation in a weight control program also improve clinical and functional outcomes for Chinese patients with schizophrenia treated with olanzapine? Proceedings of the 5th ISPOR Asia Pacific Conference; September 2-4, 2012; Taipei, Taiwan. http:/www.valueinhealthjournal.com/article/ S1098-3015\%2812\%2902106-7/abstract. Accessed May 20, 2013.

109. Chen HH, Phillips MR, Cheng H, et al. Translated and annotated version of China's new Mental Health Law. Shanghai Archives of Psychiatry. 2012;24(6):305-321.

110. Hvistendahl M. World's biggest health care system goes under the knife. Science. 2013;339(6119):505-507.

111. Soares-Weiser K, Béchard-Evans L, Lawson AH, Davis J, Ascher-Svanum H. Time to all-cause treatment discontinuation of olanzapine compared to other antipsychotics in the treatment of schizophrenia: a systematic review and meta-analysis. Eur Neuropsychopharmacol. 2013;23(2):118-125.
ClinicoEconomics and Outcomes Research

\section{Publish your work in this journal}

ClinicoEconomics \& Outcomes Research is an international, peerreviewed open-access journal focusing on Health Technology Assessment, Pharmacoeconomics and Outcomes Research in the areas of diagnosis, medical devices, and clinical, surgical and pharmacological intervention. The economic impact of health policy and health systems

\section{Dovepress}

organization also constitute important areas of coverage. The manuscript management system is completely online and includes a very quick and fair peer-review system, which is all easy to use. Visit http://www.dovepress.com/testimonials.php to read real quotes from published authors. 